\title{
Trivium
}

Revue franco-allemande de sciences humaines et sociales - Deutsch-französische Zeitschrift für Geistesund Sozialwissenschaften

$31 \mid 2020$

La culture politique de la République romaine

\section{La culture politique de la République romaine dans la recherche allemande}

\section{Martin Jehne}

Traducteur : Anthony Andurand

\section{CpenEdition}

\section{Journals}

Édition électronique

URL : https://journals.openedition.org/trivium/7242

DOI : $10.4000 /$ trivium.7242

ISBN : 1963-1820

ISSN : 1963-1820

\section{Éditeur}

Les éditions de la Maison des sciences de l'Homme

\section{Référence électronique}

Martin Jehne, «La culture politique de la République romaine dans la recherche allemande », Trivium [En ligne], 31 | 2020, mis en ligne le 30 juin 2020, consulté le 23 août 2021. URL : http:// journals.openedition.org/trivium/7242; DOI : https://doi.org/10.4000/trivium.7242

Ce document a été généré automatiquement le 23 août 2021.

\section{cc)}

Les contenus des la revue Trivium sont mis à disposition selon les termes de la Licence Creative Commons Attribution - Pas d'Utilisation Commerciale - Pas de Modification 4.0 International. 


\section{La culture politique de la République romaine dans la recherche allemande}

\section{Martin Jehne}

Traduction : Anthony Andurand

Au commencement était la Res publica amissa ${ }^{1}$. Naturellement, la recherche allemande sur la République romaine ne commence pas avec cette éminente étude de Christian Meier, et ce dernier a lui-même été fortement inspiré, notamment, par Theodor Mommsen, Matthias Gelzer, Hermann Strasburger et Alfred Heuß², dont les œuvres ont été appréciées et largement diffusées, y compris hors d'Allemagne ${ }^{3}$. Toutefois, le livre de Meier, Res publica amissa, marque tout autant un changement de perspective qu'une césure, dont l'impact persiste aujourd'hui. Dans ses efforts pour mieux comprendre les raisons pour lesquelles la République romaine a périclité, Meier a redéfini la pratique politique. Il accorda également une grande importance à ce que Gelzer appelait les "relations de fidélité et de proximité », autrement dit les relations entre patrons et clients, d'une part, et les solidarités de famille et d'amitié unissant les individus d'un même rang social, d'autre part. Il a cependant montré de manière convaincante que ce système d'échanges se modifia et n'était plus du tout, au II ${ }^{\mathrm{e}}$ et au $\mathrm{I}^{\mathrm{er}}$ siècle av. J.-C., à même de garantir le caractère fortement prévisible des élections et d'autres décisions importantes. La vie politique romaine était ainsi traversée par les efforts d'une classe pour organiser, à travers des coalitions de circonstance, le soutien nécessaire. La politique réclamait une activité permanente et une extrême agilité. Les factions plus stables que l'on avait auparavant l'habitude de déduire des relations de parenté et d'échanges n'existaient plus à la fin de la République - du moins pas autant qu'il eût été nécessaire pour pouvoir compter, dans des situations précises, sur la capacité de sa propre faction à s'imposer. Dans le même temps, l'expansion tout à fait considérable de Rome eut une profonde incidence sur les institutions, mais aussi sur le comportement des groupes et des individus. Comme l'on restait attaché, en parallèle, aux procédures forgées et au partage des pouvoirs, l'ordre de la communauté se distendit largement Meier évoque sur ce point une "extensification» de la res publica. Parmi les 
conséquences de cette extensification, Meier observa une certaine gêne, tout à fait répandue au sein des classes dirigeantes, à l'égard de l'environnement politique, sans que cette image négative fit émerger des idées de réforme plus radicales ou même une volonté de changement plus forte. Pour décrire cette situation, Meier forgea l'idée nouvelle d'une "crise sans alternative", qu'il entendait appliquer à la République d'après Sylla, en lieu et place de la notion de révolution, due à Mommsen, mais étayée sur le plan théorique par Heuß4.

2 Meier eut une série d'élèves, sans faire lui-même école, au sens strict du terme. Les thèmes et les approches de ses élèves étaient très variés. Pour la fin de la République romaine, il faut citer, en premier lieu, Kurt Raaflaub et Hinnerk Bruhns, qui proposèrent des travaux importants sur le déclenchement des guerres civiles et les clivages au sein des classes dirigeantes, inspirés sans nul doute par le fort intérêt qu'accordait Meier à l'importance de César dans la chute de la République ${ }^{5}$. Rolf Rilinger étudia le rôle du magistrat qui présidait les élections et réfuta l'idée répandue que les classes dirigeantes auraient disposé en sa personne d'un moyen, activable à tout moment, de contrer un résultat indésirable ${ }^{6}$. Il analysa également les notions concurrentes de "révolution " et de "crise sans alternative " et put faire apparaître dans ce cadre les mérites du nouveau concept de Meier ${ }^{7}$. Dans un article sur l'histoire du concept de crise, Hinnerk Bruhns, d'une part, dépassa largement le cadre des sciences de l'Antiquité et montra, d'autre part, que l'application de ce concept au contexte de la fin de la République est souvent peu raisonnée, mettant ainsi d'autant plus en relief l'importance de la lecture innovante de Meier ${ }^{8}$. Wilfried Nippel s'intéressa à l'ordre public à Rome et montra de manière convaincante comment un système normatif traditionnel, que les élites cultivées, mais aussi les simples citoyens avaient assimilé, permettait de faire en sorte, d'une part, que les rituels de la communication politique, malgré les conflits, se déroulent généralement sans anicroche; mais aussi, d'autre part, que les conflits importants ne se transforment que rarement en violence et ne visent pas a fortiori une modification fondamentale des structures politiques. Sur cette base, il put en outre montrer que la plèbe, en cas d'entorses à ces normes, était parfaitement capable d'agir spontanément et de manière autonome et n'était aucunement manipulée dans toutes ses réactions par des meneurs issus des strates supérieures ${ }^{9}$. Stimulé par les recherches de Meier sur la République, Karl-Joachim Hölkeskamp reconstitua et analysa l'émergence de la noblesse romaine durant les premiers temps et la période intermédiaire de la République. Il mit ainsi en lumière le développement de règles et de structures qui devaient être considérées, dans les temps ultérieurs, comme le noyau incontestable de l'ordre républicain ${ }^{10}$.

3 À l'époque où Christian Meier formulait sa nouvelle conception de la vie politique romaine, l'histoire sociale tendait à devenir le centre de gravité des sciences historiques en général, phénomène dont l'histoire ancienne ne restait pas exempte. D'une manière générale, on s'intéressait de plus en plus à ces groupes qui n'étaient pas habituellement placés sous les feux des projecteurs de l'histoire. L'impulsion que Jochen Martin donna à ce courant de la recherche, à travers l'attention qu'il porta à l'anthropologie historique - discipline pour laquelle il fonda à l'université de Fribourg, où il enseignait, un institut largement interdisciplinaire -, est remarquable ${ }^{11}$. Il effectua et coordonna des recherches sur l'enfance, sur la place du père dans l'Antiquité, sur les rôles de genre et plus généralement sur la famille. Il s'efforça ainsi de remonter la trace du principe même d'une anthropologie "historique", au sens propre du terme: la 
"mutation du permanent ${ }^{12}$ ». L'intérêt porté aux structures familiales et à leur importance sur le plan social demeure considérable ${ }^{13}$. De surcroît, la recherche porta une attention accrue à l'esclavage, auquel un centre de recherches était déjà dédié depuis 1950 à l'Académie de Mayence. Celui-ci avait été délibérément fondé dans le but de délaisser quelque peu, voire de contester la notion marxiste de "sociétés esclavagistes ${ }^{14} »$. Dans l'ensemble, l'histoire sociale de cette époque, souvent adossée à une démarche prosopographique, s'intéressa plutôt aux strates supérieures de l'époque impériale, pour lesquelles les inscriptions offraient un vaste matériau documentaire. Pourtant, lorsque Géza Alföldy publia en 1976 son Histoire sociale romaine (Römische Sozialgeschichte), il traita aussi, bien sûr, de la République ${ }^{15}$. L'histoire économique, longtemps laissée aux soins de quelques spécialistes, était à nouveau davantage pratiquée, évolution dans laquelle les propositions de Moses Finley jouèrent assurément un rôle important ${ }^{16}$. En Allemagne, Helmuth Schneider, en particulier, s'efforça de mettre davantage l'accent, pour l'histoire de la République elle aussi, sur les aspects économiques ${ }^{17}$.

Compte tenu de la longue tradition de droit constitutionnel associée en Allemagne aux recherches sur la République, il n'est pas étonnant que ce domaine soit demeuré un champ de travail important. Jochen Bleicken était le plus éminent représentant de ce courant. Dans son travail de thèse sur le tribunat de la plèbe au temps de la République, cependant, il insistait déjà, en parallèle des éléments relevant du droit constitutionnel, sur le rôle politique de cette magistrature et de ses détenteurs, en soulignant son lien avec le Sénat et l'attitude largement coopérative des tribuns à cette époque, envisagés comme un phénomène extra-juridique ${ }^{18}$. Dans son étude sur la législation romaine, il classa les décisions des assemblées populaires romaines selon qu'elles se rattachent à des circonstances particulières ou qu'elles revêtent un caractère normatif. Il envisagea le flot de lois qui marque la fin de la République comme un durcissement, dans lequel les liens personnels extra-juridiques de nature égalitaire et hiérarchique, cœur de l'ordre aristocratique, subissent de plus en plus la pression de prescriptions devenues impersonnelles et avec lesquelles ils entretiennent une relation conflictuelle ${ }^{19}$. Christian Meier a toutefois critiqué l'opposition entre ordre juridique et ordre social ${ }^{20}$. En outre, dans l'intervalle, Christoph Lundgreen a montré, au travers de la distinction entre règles et principes, comment la République romaine fonctionnait en partie sur la base de normes souples, qui pouvaient être écartées dans certains cas, sans perdre leur caractère normatif ${ }^{21}$.

Deux ouvrages magistraux parurent en France en 1976: Le métier de citoyen de Claude Nicolet et Le pain et le cirque de Paul Veyne ${ }^{22}$. Dans l'une et l'autre de ces études, les relations particulières qu'entretiennent le peuple et les élites à Rome occupent une place centrale, mais la matière principale et l'approche diffèrent fortement. Pour autant, on peut décrire ces perspectives et ces clés interprétatives, je pense, comme un communicative turn dans les recherches sur l'histoire romaine ${ }^{23}$. Ces ouvrages connurent une diffusion en Allemagne, mais celle-ci fut limitée, même si le livre de Veyne comme d'autres livres de cet historien - fut traduit en allemand et eut ainsi de meilleures chances que l'ouvrage de Nicolet, dont la version américaine fut probablement davantage utilisée que la version originale en français. Jean-Michel David, Hinnerk Bruhns et Wilfried Nippel ont favorisé le développement des échanges entre la recherche française et la recherche allemande sur la République romaine. Ils organisèrent un colloque à Strasbourg, lors duquel furent présentées des communications portant sur des aspects essentiels de la fin de la République et 
commentées, dans chaque cas, par un participant ou une participante de l'autre pays ${ }^{24}$. Les différences et les convergences, mais plus encore les points de contact se firent à cette occasion plus évidents.

6 La recherche sur la République romaine, en général et peut-être plus particulièrement en Allemagne, reçut une impulsion importante des travaux menés par Fergus Millar depuis 1984. Millar s'était jusqu'alors surtout intéressé à l'époque impériale et à l'Antiquité tardive. Son ouvrage systématique sur l'empereur romain comme figure de pouvoir constituait un classique, qui compte toujours parmi les ouvrages fondamentaux ${ }^{25}$. Il s'intéressa ensuite à la République et développa dans une série d'articles sa thèse centrale, à savoir que la République romaine, à rebours de la vision courante selon laquelle ce système politique devait être classé parmi les systèmes aristocratiques ou oligarchiques, constituait fondamentalement une démocratie ${ }^{26}$. Son jugement se fonde sur le fait incontestable qu'à Rome, la politique devait être expliquée et validée devant les citoyens et que les décisions obligatoires devaient recueillir l'assentiment de tous les citoyens présents dans les assemblées. La nouvelle interprétation de Millar suscita l'adhésion et la contradiction ${ }^{27}$, mais pour les historiens et les historiennes dont le regard sur la République romaine était en Allemagne inspiré par la Res publica amissa de Meier, cette classification se révéla peu éclairante. Lors du congrès des historiens allemands (Deutscher Historikertag) de 1994, à Leipzig, se tint une section animée par Egon Flaig, Karl-Joachim Hölkeskamp et moi-même, sous le titre "Démocratie à Rome? Le rôle du peuple dans la vie politique de la République romaine ». Les contributions furent publiées l'année suivante sous la forme d'un volume collectif ${ }^{28}$. La critique des vues de Millar s'appuya surtout, à cette occasion, sur les catégories et les concepts issus des recherches portant sur la culture politique. Ce thème et cette approche tendirent depuis lors à occuper le devant de la scène dans la recherche allemande ${ }^{29}$.

7 Dans son ouvrage sur les usurpations du début de l'époque impériale, Egon Flaig avait déjà décrit une certaine forme du rapport au peuple romain dans les assemblées comme un rituel consensuel ${ }^{30}$. Il suivait ainsi la distinction fondamentale, formulée dans le cadre de la recherche sur la culture politique, entre le contenu et la forme de la politique, qui permet d'ouvrir le regard sur le fait qu'il n'est pas du tout rare que les systèmes politiques qui se réclament de la participation de la communauté citoyenne fassent appel à des actes de communication ritualisés dans les assemblées du « peuple ». Les élites confirment ainsi l'importance du peuple, cependant que le peuple valide les décisions recommandées en amont par les élites. S'il existe en plus - comme c'est le cas dans la Rome républicaine - une structure pour l'élection des personnes dans laquelle ce ne sont pas des concepts politiques, mais seulement des personnalités plus ou moins honorables qui s'offrent au choix, desquelles on n'avait généralement à attendre aucune différence importante dans la gestion de la charge qui leur était confiée, alors le choix concret effectué lors de l'élection ne revêt de toute façon qu'une importance relativement limitée. ${ }^{31}$ C'est sur ces présupposés, dont Karl-Joachim Hölkeskamp a résumé succinctement les sources dans la contribution proposée dans ce numéro de Trivium $^{32}$, que se développèrent par la suite les analyses du fonctionnement de la République romaine. Dans l'ouvrage La politique ritualisée (Ritualisierte Politik) publié en 2003, dans lequel Egon Flaig a réuni de manière systématique ses recherches sur la République romaine, les diverses suggestions dues aux sociologues français et aux historiens issus en Allemagne de la recherche sur la République apparaissent clairement ${ }^{33}$. Parmi les formes ritualisées de la communication que Flaig a analysées 
dans son ouvrage, les gestes de coercition sont selon lui mis en exergue dans ce contexte - Christoph Degelmann a aujourd'hui consacré au geste du deuil une monographie dédiée ${ }^{34}$. Jan Timmer a montré que les procédures et les rituels du Sénat incorporaient largement des éléments de consensus ${ }^{35}$.

Depuis la fin des années 1990, la question du rôle du peuple romain dans la vie politique de l'époque républicaine constituait un objet central des recherches sur la République, sur le plan national comme sur le plan international. Alors que la recherche anglophone épousait en partie les conceptions développées par Millar, on demeurait plutôt sceptique en Allemagne. Une part essentielle des analyses se focalisaient sur les contiones, au double sens d'assemblée et de discours tenu devant l'assemblée, et donc en tant que performance des orateurs ${ }^{36}$. Une nouvelle tendance s'introduisit dans le débat, lorsque Henrik Mouritsen développa la thèse selon laquelle les assemblées romaines ne devaient pas être envisagées comme un élément démocratique, non seulement au motif qu'elles ne remplissaient au fond qu'une fonction d'approbation, mais aussi parce que le peuple, autrement dit les citoyens les moins aisés, n'y était pas présent. Dans cette lecture, on comptait principalement, dans l'assistance des contiones, des partisans issus de la leisure class $^{37}$. Contre cette théorie, qui constitue le pôle opposé des conceptions de Millar, se manifesta également une certaine résistance, en particulier au nom de l'argument selon lequel une condition économique modeste ne signifiait pas, dans les sociétés prémodernes, que les citoyens concernés ne trouvaient pas le temps d'écouter des orateurs politiques - d'autant qu'il était courant, dans les contiones, que les orateurs flattent les présents et soulignent la souveraineté du peuple en matière de décisions ${ }^{38}$. Pourtant, il existe aussi un courant soucieux de ne pas sous-estimer l'influence du peuple ordinaire ni de surestimer l'idée d'un contrôle par le haut ${ }^{39}$. Comme élément d'une analyse plus détaillée des formes de communication entre les strates supérieures et inférieures, les procédures de vote complexes et les activités de campagne des candidats, dont l'ambitus - les manœuvres, considérées comme illégales, visant à influencer le vote, notamment à travers l'achat des voix -, furent largement étudiées ${ }^{40}$.

9 À travers l'attention portée à la communication entre les élites et les simples citoyens, d'autres lieux de rencontre gagnèrent en importance. Egon Flaig a souligné que la reprise, au miroir de l'actualité, de passages tirés de pièces de théâtre par les spectateurs réunis revêtait une certaine importance en tant que moyen d'expression politique $^{41}$. Il s'est en outre intéressé à la pompa funebris ${ }^{42}$. Hans Beck et plus largement Karl-Joachim Hölkeskamp se sont emparés de ce qu'on appelle le spatial turn et ont analysé les différents types de processions, en lien avec le paysage mémoriel de Rome ${ }^{43}$. Uwe Walter, en particulier, s'est fortement consacré à la culture mémorielle romaine et aux formes concrètes du rapport au passée ${ }^{44}$. Wolfgang Blösel et Michael Stemmler, parmi d'autres, ont étudié l'importance accordée au mos maiorum, tandis que la question de la tolérance aux écarts a été traitée par Jani Kirov ${ }^{45}$. Ernst Baltrusch a étudié la censure, dans son attachement à la préservation de la tradition; Rene Pfeilschifter a abordé le census comme un rituel d'intégration et expliqué, à partir de cette perspective, pour quelles raisons le census dépérit peu ou prou dès avant la fin de la République. Du fait de l'assouplissement des règles de représentation, en effet, les membres des strates dirigeantes n'étaient déjà plus présents lors du census et se contentaient d'y envoyer leurs intendants, de sorte que le peuple dans son ensemble n'y apparaissait plus et que le sentiment de communauté ne pouvait plus s'y développer ${ }^{46}$. Tanja Itgenshorst a consacré une analyse fondamentale au triomphe 
romain, dont elle a éclairé la structure rituelle, en se concentrant sur les sources de l'époque républicaine ${ }^{47}$.

10 En 2004, Karl-Joachim Hölkeskamp publia l'ouvrage Reconstruire une République. La "culture politique» de la Rome antique et la recherche des dernières décennies (Rekonstruktionen einer Republik. Die politische Kultur des antiken Rom und die Forschungen der letzten Jahrzehnte). Il fut traduit en français dès 2008, en 2010 en anglais (dans une version fortement augmentée), en italien également en 2016 et en espagnol en 2019. Toutes ces éditions, dans les langues qu'utilisent habituellement les sciences de l'Antiquité pour communiquer, suffisent à montrer l'ample succès de ce nouveau jalon de référence de la recherche, qui offre un vaste inventaire et met clairement en évidence, dans le même temps, combien les concepts issus des recherches sur la culture politique se sont révélés féconds pour la compréhension de la République romaine. Depuis lors, Hölkeskamp a continué d'approfondir cette approche dans le cadre d'articles programmatiques, portant par exemple sur les dimensions performatives et spatiales des processions ${ }^{48}$, ou encore sur la compétition et l'esprit agonistique ${ }^{49}$. Marian Nebelin a consacré une étude, remarquable sur le plan conceptuel et analytique, à la compétition sur la scène sénatoriale et à son effondrement à la fin de la République $^{50}$.

11 L'ouvrage pionnier de Christian Meier, Res publica amissa, a incité la recherche à s'interroger, à nouveaux frais et de manière approfondie, sur le fonctionnement et le succès de la République, mais aussi et surtout sur l'étendue et les raisons de sa faillite, du moins dans les dernières années de son existence. Dans la nouvelle introduction de la deuxième édition de l'ouvrage, publiée en 1980 - dont le présent numéro offre une version abrégée traduite en français -, Meier a donné encore une fois de nouvelles impulsions. Dans cette nouvelle réflexion, en effet, de nombreux éléments, qui étaient encore relevés de manière relativement descriptive dans la première édition, sont plus fortement marqués sur le plan théorique. En outre, en 2015, Meier a rassemblé en détail ses conceptions relatives à "l'ordre de la République romaine ${ }^{51}$ ". Ces recherches poussées sur le rôle de César, qui trouvèrent leur point d'orgue dans la volumineuse biographie $^{52}$ parue en 1982, proposèrent notamment, parmi d'autres éléments, un approfondissement de la thèse d'une "crise sans alternative ». Pour autant, Meier perçut en César, qui finit assurément par s'ériger en autocrate, un grand désemparement quant à ce qu'il pouvait bien faire de la position de domination qu'il avait acquise : en fin de compte, l'absence d'alternative qui avait caractérisé l'ancienne République se poursuivait. De surcroit, Meier avait collaboré au développement de la théorie des processus historiques et rattaché l'idée d'une crise sans alternative, envisagée comme processus autonome, à un concept abstrait ${ }^{53}$. Le sentiment d'une crise éprouvé à la fin de la République revêtait ainsi une actualité accrue. Après que Klaus Martin Girardet et Gustav Adolf Lehmann eurent défendu la capacité de la République à se réformer ${ }^{54}$, Monika Bernett, une élève de Meier, analysa la réflexion sur la crise de Cicéron $^{55}$. Dans les actes d'un colloque consacré à l'œuvre de Christian Meier, elle détailla ensuite l'usage de la notion de "crise sans alternative » dans l'œuvre de ce dernier ${ }^{56}$. Aloys Winterling, un autre élève de Meier, qui s'est principalement intéressé à l'époque impériale, identifia chez Meier, dans ce même volume, trois acceptions distinctes de la notion de crise. Il désigna l'une d'elles, décrite comme un fort sentiment de carence répondant à l'accroissement frénétique des activités familières, au moyen du concept d'«involution », emprunté à l'ethnologie ${ }^{57}$. Ce diagnostic a été validé par Wolfgang Blösel sous un tout autre aspect. Ce dernier a remarqué que, plus 
particulièrement dans la République d'après Sylla, un grand nombre de consuls et de préteurs refusaient de se voir confier une province, renonçant ainsi à la possibilité d'acquérir la gloire militaire et des avantages matériels ${ }^{58}$. L'idée semble avoir prédominé que l'on ne pouvait s'éloigner trop longtemps de l'activité politique trépidante de Rome sans perdre en influence. À l'inverse, Jürgen von Ungern-Sternberg a constaté un relâchement de la pression liée à l'Empire et à la politique extérieure en général, moyennant quoi les aristocrates romains se virent dispenser de l'obligation de toujours s'entendre, au nom de la défense et de l'extension de l'Empire. Au contraire, ils pouvaient désormais s'adonner librement à leurs luttes de pouvoir intestines et négliger les préoccupations des citoyens ordinaires ${ }^{59}$. Uwe Walter a examiné, dans le cadre d'une réflexion plus fondamentale, la part de la contingence que revêtent ces évolutions ${ }^{60}$. Il a lui aussi rédigé récemment un important ouvrage de synthèse ${ }^{61}$.

Au diagnostic de Christian Meier, selon lequel la domination de César n'offrait aucune perspective pour surmonter la crise ${ }^{62}$, Mischa Meier a opposé une nouvelle approche. En distinguant le discours et le cadre d'action, il a constaté, s'agissant de César, que si la monarchie, envisagée comme un moyen de surmonter la crise républicaine, était selon lui déjà présente dans le cadre d'action, il demeurait pour longtemps encore impossible de prononcer ce nom dans le discours ${ }^{63}$. En outre, Jan Timmer a introduit la catégorie de la « confiance » dans les débats sur la crise de la République et discuté de la perte de confiance comme une possible composante du scénario de la chute - bien qu'il soit difficile, comme il le souligne lui-même, de déterminer, au regard des sources, si les guerres civiles, depuis les années $40 \mathrm{du} \mathrm{I}^{\mathrm{er}}$ siècle avant J.-C., était plus une conséquence ou une cause de la perte de confiance ${ }^{64}$.

Dans le prolongement des riches discussions qui ont animé les dernières décennies, la République romaine est restée d'actualité dans les recherches en histoire ancienne menées en Allemagne, peut-être dans une moindre mesure, toutefois, qu'il y a encore quelques années. En revanche, il existe une série d'ouvrages sur les débuts de la période impériale, largement inspirés, eux aussi, des approches issues de la recherche sur la culture politique. L'étude d'Andreas Klingenberg sur la relégation sociale à l'époque impériale précède l'intérêt accru pour les perdants de la République ${ }^{65}$. Le Sénat romain de l'époque impériale est abordé par Isabelle Künzer, qui met en exergue une culture spécifique de la concurrence, ainsi que par Simone Blochmann, qui analyse résolument - selon le sous-titre de son ouvrage - «la culture politique du Sénat au début de l'époque impériale ${ }^{66} »$. Katja Kröss a étudié le rôle du peuple à l'époque impériale, un thème depuis longtemps présent dans les recherches sur la culture politique de la République $^{67}$.

14 Si l'on spécule sur la manière dont ces perspectives et ces approches continueront de se développer, on peut à coup sûr s'attendre à ce que les normes de conduite propres à culture romaine et leurs lentes évolutions continueront de susciter l'intérêt, peut-être même dans un rapport plus étroit aux arènes, c'est-à-dire aux espaces, aux genres et aux règles qui sous-tendent les communautés de communication, assimilées par les membres d'une culture donnée. Ainsi étroitement mises en relation, les représentations que suscitent les normes et les diverses obligations qu'elles véhiculent demeureront sans nul doute un sujet d'actualité, et les nouvelles discussions sur l'étaticité, qui mobilisent des catégories intéressantes, car décorrélées de l'État moderne, s'avéreront fécondes pour l'Antiquité68. Il ne reste donc qu'à espérer, en fin 
de compte, que surgissent de multiples pistes inattendues et pour cette raison d'autant plus excitantes - comme ce fut le cas en 1966, avec la parution de Res publica amissa.

\section{BIBLIOGRAPHIE}

Alföldy, G. (1976) : Römische Sozialgeschichte, Wiesbaden ; 2 éd. $1979 ; 3^{\mathrm{e}}$ éd. 1984.

Alföldy, G. (2011) : Römische Sozialgeschichte, $4^{\mathrm{e}}$ éd. révisée et actualisée, Stuttgart.

Angius, A. (2018) : La Repubblica delle opinioni. Informazione politica e partecipazione popolare a Roma tra II e I secolo a.C., Milan.

Baltrusch, E. (1989) : Regimen morum. Die Reglementierung des Privatlebens der Senatoren und Ritter in der römischen Republik und frühen Kaiserzeit, Munich.

Beck, H. (2005) : « Züge in die Ewigkeit. Prozessionen durch das republikanische Rom », Göttinger Forum für Altertumswissenschaft, 8, p. 73-104.

Beck, H. (2019) : Pecuniam inlargibo tibi. Wahlbestechung und Wahlniederlage in der mittleren römischen Republik, in : Hölkeskamp, K.-J. / Beck, H. (éd.) : Verlierer und Aussteiger in der « Konkurrenz unter Anwesenden ». Agonalität in der politischen Kultur des antiken Rom, Stuttgart, p. 31-53.

Bernett, M. (1995) : Causarum cognitio. Ciceros Analysen zur politischen Krise der späten römischen Republik (Palingenesia, 31), Stuttgart.

Bernett, M. (2008) : « Krisenbewußtsein der späten römischen Republik », in : Bernett / Nippel / Winterling (2008), p. 161-179.

Bernett, M. / Nippel, W. / Winterling, A. (éd.) (2008) : Christian Meier zur Diskussion. Autorenkolloquium am Zentrum für Interdisziplinäre Forschung in Bielefeld, Stuttgart.

Bleicken, J. (1955) : Das Volkstribunat der klassischen Republik. Studien zu seiner Entwicklung zwischen 287 und 133 v.Chr., Munich, $2^{\mathrm{e}}$ éd. 1968.

Bleicken, J. (1975) : Lex publica. Gesetz und Recht in der römischen Republik, Berlin / New York.

Blochmann, S. (2017) : Verhandeln und entscheiden. Politische Kultur im Senat der frühen Kaiserzeit (Historia Einzelschriften, 245), Stuttgart.

Blösel, W. (2000) : « Die Geschichte des Begriffs mos maiorum von den Anfängen bis zu Cicero », in : Linke, B. / Stemmler, M. (éd.) : Mos maiorum. Untersuchungen zu den Formen der Identitätsstiftung und Stabilisierung in der römischen Republik (Historia Einzelschriften, 141), Stuttgart, p. 25-97.

Blösel, W. (2011) : « Die Demilitarisierung der römischen Nobilität von Sulla bis Caesar », in : Blösel, W. / Hölkeskamp, K.-J. (éd.) : Von der militia equestris zur militia urbana. Prominenzrollen und Karrierefelder im antiken Rom (actes du colloque international, 16-18 mai, Université de Cologne), Stuttgart, p. 55-80.

Blösel, W. (2016) : « Provincial Commands and Money in the Late Roman Republic », in : Beck, H. / Jehne, M. / Serrati, J. (éd.) : Money and Power in the Roman Republic (Collection Ltomus, 355), Bruxelles, p. 68-81. 
Blösel, W. (en préparation) : Imperia extraordinaria liberae rei publicae - Studien zur Demilitarisierung der römischen Nobilität, thèse d'habilitation, Cologne.

Bruhns, H. (1978) : Caesar und die römische Oberschicht in den Jahren 49-44 v.Chr. Untersuchungen zur Herrschaftsetablierung im Bürgerkrieg (Hypomnemata, 55), Göttingen.

Bruhns, H. (1997) : « Zur Wirtschaftsgeschichte der römischen Republik in der deutschen Forschung. Kommentar zum Beitrag von Jean Andreau », in : Bruhns / David / Nippel (1997), p. 185-193.

Bruhns, H. (2003) : «Crise de la République romaine ? Quelle crise ? ", in : Franchet d'Espèrey, S. / Fromentin, V. / Gotteland, S. / Roddaz, J.-M. (éd.) : Fondements et crises du pouvoir (AusoniusPublications Études, 9), Bordeaux, p. 365-378.

Bruhns, H. / David, J.-M. / Nippel, W. (éd.) (1997) : Die späte römische Republik - La fin de la République romaine. Un débat franco-allemand d'histoire et d'historiographie (Collection de l'École française de Rome, 235), Paris / Rome.

Burckhardt, L. A. (1990) : « The Political Elite of the Roman Republic: Comments on Recent Discussion of the Concepts nobilitas and homo novus ", Historia, 39, p. 77-99.

Degelmann, C. (2018) : Squalor. Symbolisches Trauern in der Politischen Kommunikation der Römischen Republik und Frühen Kaiserzeit (Potsdamer Altertumswissenschaftliche Beiträge, 61), Stuttgart.

Finley, M. I. (1973) : The Ancient Economy, Berkeley / Los Angeles ; $2^{\mathrm{e}}$ éd. 1984 ;

trad. allemande : Die antike Wirtschaft, trad. par A. Wittenburg, Munich $1977 ; 3^{\mathrm{e}}$ éd. 1993.

Flaig, E. (1992) : Den Kaiser herausfordern. Die Usurpation im Römischen Reich, Francfort-sur-le-Main / New York.

Flaig, E. (1993) : « Politisierte Lebensführung und ästhetische Kultur. Eine semiotische Untersuchung am römischen Adel », Historische Anthropologie, 1, p. 193-217.

Flaig, E. (1994) : « Repenser le politique dans la République romaine », Actes de la Recherche en Sciences sociales, 105, p. 13-25.

Flaig, E. (1995a) : « Entscheidung und Konsens. Zu den Feldern der politischen Kommunikation zwischen Aristokratie und Plebs », in : Jehne (1995), p. 77-127.

Flaig, E. (1995b) : « Die Pompa Funebris. Adlige Konkurrenz und annalistische Erinnerung in der Römischen Republik », in : Oexle, O.G. (éd.) : Memoria als Kultur, Göttingen, p. 115-148.

Flaig, E. (1997) : « Zwingende Gesten in der römischen Politik », in : Chvojka, E. / van Dülmen, R. / Jung, V. (éd.) : Neue Blicke. Historische Anthropologie in der Praxis, Vienne / Cologne / Weimar, p. 33-50.

Flaig, E. (1998) : « War die römische Volksversammlung ein Entscheidungsorgan? Institution und soziale Praxis », in : Blänkner, R. / Jussen, B. (éd.) : Institutionen und Ereignis. Über historische Praktiken und Vorstellungen gesellschaftlichen Ordnens, Göttingen, p. 49-73.

Flaig, E. (2003) : Ritualisierte Politik. Zeichen, Gesten und Herrschaft im Alten Rom (Historische Semantik, 1), Göttingen.

Gelzer, M. (1912) : Die Nobilität der römischen Republik, Leipzig / Berlin ; reproduit in : Gelzer, M. (1962) : Kleine Schriften, vol. I, Wiesbaden, p. 17-135.

Girardet, K. M. (1983) : Die Ordnung der Welt. Ein Beitrag zur philosophischen und politischen Interpretation von Ciceros Schrift de legibus (Historia Einzelschriften, 42), Wiesbaden. 
Girardet, K. M. (2018) : « Hat Cicero, ohne es zu bemerken, die Einführung der Monarchie in Rom vorgeschlagen?», Hermes, 146, p. 116-121.

Harders, A.-C. (2008) : Suavissima Soror. Untersuchungen zu den Bruder-Schwester-Beziehungen in der römischen Republik (Vestigia, 60), Munich.

Heuß, A. (1956) : « Der Untergang der römischen Republik und das Problem der Revolution », Historische Zeitschrift, 182, p. 1-28.

Heuß, A. (1973) : « Das Revolutionsproblem im Spiegel der antiken Geschichte », Historische Zeitschrift, 216, p. 1-72.

Hölkeskamp, K.-J. (1987) : Die Entstehung der Nobilität. Studien zur sozialen und politischen Geschichte der Römischen Republik im 4. Jhdt. v. Chr., Stuttgart ; $2^{\mathrm{e}}$ éd. augmentée 2011.

Hölkeskamp, K.-J. (1995) : « Oratoris maxima scaena. Reden vor dem Volk in der politischen Kultur der Republik », in : Jehne (1995), p. 11-49;

révisé et complété in : Hölkeskamp, K.-J. (2004) : Senatus Populusque Romanus. Die politische Kultur der Republik - Dimensionen und Deutungen, Stuttgart, p. 219-256.

Hölkeskamp, K.-J. (2004) : Rekonstruktionen einer Republik. Die politische Kultur des antiken Rom und die Forschung der letzten Jahrzehnte (Historische Zeitschrift Beihefte [nouvelle série], 38), Munich ; trad. en français : 2008 ; en anglais : 2010 ; en italien : 2016 ; en espagnol : 2019.

Hölkeskamp, K.-J. (2006) : « Konsens und Konkurrenz. Die politische Kultur der römischen Republik in neuer Sicht », Klio, 88, p. 360-396;

réimpression révisée in Hölkeskamp (2017) : Libera Res Publica. Die politische Kultur des antiken Rom - Positionen und Perspektiven, Stuttgart, p. 123-161.

Hölkeskamp, K.-J. (2008) : « Hierarchie und Konsens. Pompae in der politischen Kultur der römischen Republik », in : Arweiler, A. H. / Gauly, B. M. (éd.) : Machtfragen. Zur kulturellen Repräsentation und Konstruktion von Macht in Antike, Mittelalter und Neuzeit, Stuttgart, p. 79-126 ; réimpression révisée in : Hölkeskamp, K.-J. (2017), Libera Res Publica. Die politische Kultur des antiken Rom - Positionen und Perspektiven, Stuttgart, p. 189-236.

Hölkeskamp K.-J. (2011) : « Self-serving Sermons. Oratory and the self-construction of the republican aristocrat », in : Smith, C. / Corvino, R. (éd.) : Praise and Blame in Roman Republican Rhetoric, Swansea, p. 17-34.

Hölkeskamp, K.-J. (2013a) : « Friends, Romans, countrymen. Addressing the Roman People and the Rhetoric of Inclusion ", in : Steel, C. / van der Blom, H. (éd.) : Community and Communication. Oratory and Politics in Republican Rome, Oxford, p. 11-28.

Hölkeskamp, K.-J. (2013b) : « Concordia contionalis. Die rhetorische Konstruktion von Konsens in der römischen Republik », in : Flaig, E. / Müller-Luckner, E. (éd.) : Genesis und Dynamiken der Mehrheitsentscheidung, Munich, p. 101-128;

réimpression révisée in : Hölkeskamp, K.-J. (2017) : Libera Res Publica. Die politische Kultur des antiken Rom - Positionen und Perspektiven, Stuttgart, p. 163-188.

Hölkeskamp, K.-J. (2014) : « Konkurrenz als sozialer Handlungsmodus - Positionen und Perspektiven der historischen Forschung ", in : Jessen, R. (éd.) : Konkurrenz in der Geschichte. Praktiken - Werte - Institutionalisierungen, Francfort-sur-le-Main / New York, p. 33-57.

Hölkeskamp, K.-J. (2015) : « "Performative turn” meets "spatial turn”. Prozessionen und andere Rituale in der neueren Forschung ", in : Boschung, D. / Hölkeskamp, K.-J. / Sode, C. (éd.) : Raum und Performanz. Rituale in Residenzen von der Antike bis 1815, Stuttgart, p. 15-74. 
Hölkeskamp, K.-J. (2017) : « Politische Kultur - Karriere eines Konzepts. Ansätze und Anwendungen am Beispiel der römischen Republik », in : Haake, M. / Harders, A.-C. (éd.) : Politische Kultur und soziale Struktur der römischen Republik. Akten eines Kolloquiums aus Anlass des 70. Todestags von Friedrich Münzer, Stuttgart, p. 457-495; réimpression révisée in : Hölkeskamp, K.-J. (2017) : Libera Res Publica. Die politische Kultur des antiken Rom - Positionen und Perspektiven, Stuttgart, p. 73-105.

Hölkeskamp, K.-J. (2019a) : “ "Cultural Turn” oder gar Paradigmenwechsel in der Althistorie? Die politische Kultur der römischen Republik in der neueren Forschung », Historische Zeitschrift, 309, p. 1-35.

Hölkeskamp, K.-J. (2019b) : « Verlierer in der “Konkurrenz unter Anwesenden”. Agonalität in der politischen Kultur der römischen Republik », in : Hölkeskamp, K.-J. / Beck, H. (éd.) : Verlierer und Aussteiger in der « Konkurrenz unter Anwesenden ». Agonalität in der politischen Kultur des antiken Rom, Stuttgart, p. 11-29.

Itgenshorst, T. (2005) : Tota illa pompa. Der Triumph in der römischen Republik, Göttingen.

Jehne, M. (1987) : Der Staat des Dictators Caesar, Cologne / Vienne.

Jehne, M. (éd.) (1995) : Demokratie in Rom? Die Rolle des Volkes in der Politik der römischen Republik, Stuttgart.

Jehne, M. (1995a) : « Einführung: Zur Debatte um die Rolle des Volkes in der römischen Politik », in : Jehne (1995), p. 1-9.

Jehne, M. (1995b) : « Die Beeinflussung von Entscheidungen durch “Bestechung”: Zur Funktion des ambitus in der römischen Republik», in : Jehne (1995), p. 51-76.

Jehne, M. (2000) : « Jovialität und Freiheit. Zur Institutionalität der Beziehungen zwischen Oberund Unterschichten in der römischen Republik ", in : Linke, B. / Stemmler, M. (éd.) : Mos maiorum. Untersuchungen zu den Formen der Identitätsstiftung und Stabilisierung in der römischen Republik (Historia Einzelschriften, 141), Stuttgart, p. 207-235.

Jehne, M. (2001/2003a) : «Integrationsrituale in der römischen Republik. Zur einbindenden Wirkung der Volksversammlungen », in : Urso, G. (éd.) : Integrazione, mescolanza, rifiuto. Incontri di popoli, lingue e culture in Europa dall'Antichità all'Umanesimo, Atti del convegno internazionale, Cividale del Friuli, 21-23 septembre 2000, Rome, 2001, p. 89-113 (légèrement révisé in Hölkeskamp, K.-J. / Rüsen, J. / Stein-Hölkeskamp, E. / Grütter, H. Th. [éd.] [2003] : Sinn (in) der Antike. Orientierungssysteme, Leitbilder und Wertkonzepte im Altertum, Mayence, p. 279-297).

Jehne, M. (2003b) : « Krisenwahrnehmung und Vorschläge zur Krisenüberwindung bei Cicero », in : Franchet d'Espèrey, S. / Fromentin, V. / Gotteland, S. / Roddaz, J.-M. (éd.) : Fondements et crises du pouvoir (Ausonius-Publications Études, 9), Bordeaux, p. 379-396.

Jehne, M. (2006a) : « Who Attended Roman Assemblies? Some Remarks on Political Participation in the Roman Republic », in : Marco Simón, F. / Pina Polo, F. / Remesal Rodríguez, J. (éd.) : Repúblicas y ciudadanos: modelos de participación cívica en el mundo antiguo (Instrumenta, 21), Barcelone, p. 221-234.

Jehne, M. (2006b) : « Methods, Models, and Historiography », in : Rosenstein, N. / Morstein-Marx, R. (éd.) : A Companion to the Roman Republic, Oxford, p. 3-28.

Jehne, M. (2008) : « Christian Meier und Iulius Caesar, oder: Das Faszinosum des Außenseiters », in : Bernett / Nippel / Winterling (2008), p. 201-217.

Jehne, M. (2009a) : « Caesars Alternative(n). Das Ende der römischen Republik zwischen autonomem Prozeß und Betriebsunfall », in : Hölkeskamp, K.-J. (éd.) : Eine politische Kultur (in) der 
Krise? Die «letzte Generation » der römischen Republik (Schriften des Historischen Kollegs, 73), Munich, p. 141-160.

Jehne, M. (2009b) : « Le système électoral des Romains et le désespoir des candidats », RHD, 87, p. 498-501.

Jehne, M. (2010) : « Die Dominanz des Vorgangs über den Ausgang. Struktur und Verlauf der Wahlen in der römischen Republik », in : Dartmann, Chr. / Wassilowsky, G. / Weller, Th. (éd.) : Technik und Symbolik vormoderner Wahlverfahren (Historische Zeitschrift Beiheft, 52), Munich, p. 17-34.

Jehne, M. (2011) : « Blaming the people in front of the people. Restraint and outbursts of orators in Roman contiones ", in : Smith, Chr. / Covino, R. (éd.) : Praise and Blame in Roman Republican Rhetoric, Swansea, p. 111-125.

Jehne, M. (2013a) : «Feeding the plebs with words. The significance of senatorial public oratory in the small world of Roman politics ", in : Steel, C. / van der Blom, H. (éd.) : Community and Communication. Oratory and Politics in Republican Rome, Oxford, p. 49-62.

Jehne, M. (2013b) : « Politische Partizipation in der römischen Republik », in : Reinau, H. / Ungern-Sternberg, J. v. (éd.) : Politische Partizipation. Idee und Wirklichkeit von der Antike bis in die Gegenwart (Colloquium Rauricum, 13), Berlin / Boston, p. 103-144.

Jehne, M. (éd.) (2015) : Demokratie in Rom? Die Rolle des Volkes in der Politik der römischen Republik (Historia Einzelschriften, 96), Stuttgart.

Jehne, M. (2020) : « Die Chance, eine Alternative zu formulieren, und die Chance, eine Alternative zu verwirklichen. Das Sagbare und das Machbare im republikanischen und augusteischen Rom », in : Nebelin, M. / Tiersch, C. (éd.) : Semantische Kämpfe zwischen Republik und Prinzipat? Kontinuität und Transformation der politischen Sprache in Rom (Historische Semantik, 31), Göttingen, p. 73-101 (sous presse).

Karataş, S. (2019) : Zwischen Bitten und Bestechen. Ambitus in der politischen Kultur der römischen Republik - Der Fall des Cn. Plancius (Hermes Einzelschriften, 115), Stuttgart.

Kirov, J. (2010) : « Der Umgang mit Abweichungen in der römischen Republik », Historische Zeitschrift, 290, p. 297-320.

Klingenberg, A. (2011) : Sozialer Abstieg in der römischen Kaiserzeit. Risiken in der Oberschicht in der Zeit von Augustus bis zum Ende der Severer, Paderborn.

Knopf, F. (2018) : Die Partizipationsmotive der plebs urbana im spätrepublikanischen Rom, Berlin.

Kröss, K. (2017) : Die politische Rolle der stadtrömischen Plebs in der Kaiserzeit (Impact of Empire, 24), Leyde / Boston.

Künzer, I. (2016) : Kulturen der Konkurrenz. Untersuchungen zu einem senatorischen Interaktionsmodell an der Wende vom ersten zum zweiten Jahrhundert n.Chr. (Antiquitas I, 68), Bonn.

Laser, G. (1997) : Populo et scaenae serviendum est. Die Bedeutung der städtischen Masse in der Späten Römischen Republik, Trèves.

Lehmann, G. A. (1980) : Politische Reformvorschläge in der Krise der späten römischen Republik. Cicero De legibus III und Sallusts Sendschreiben an Caesar, Meisenheim.

Linke, B. (1998) : « Die agnatio. Ein römischer Sonderweg in der sozialen Organisation », Historische Anthropologie, 6, p. 104-131. 
Linke, B. (2006) : « Bürger ohne Staat? Die Integration der Landbevölkerung in der römischen Republik », in : Jehne, M. / Pfeilschifter, R. (éd.) : Herrschaft ohne Integration? Rom und Italien in republikanischer Zeit, Francfort-sur-le-Main, p. 65-94.

Linke, B. (2014) : « Die Väter und der Staat. Die Grundlagen der aggressiven Subsidiarität in der römischen Gesellschaft », in : Lundgreen, C. (éd.) : Staatlichkeit in Rom?, Stuttgart, p. 65-90.

Lundgreen, C. (2011) : Regelkonflikte in der römischen Republik. Geltung und Gewichtung von Normen in politischen Entscheidungsprozessen (Historia Einzelschriften, 221), Stuttgart.

Lundgreen, C. (éd.) (2014) : Staatlichkeit in Rom? Diskurse und Praxis (in) der römischen Republik (Staatsdiskurse, 28), Stuttgart.

Lundgreen, C. (2014a) : « Staatsdiskurse in Rom? Staatlichkeit als analytische Kategorie für die römische Republik », in : Lundgreen (2014), p. 15-61.

Martin, J. (1982) : « Das Institut für Historische Anthropologie », Saeculum, 33, p. 375-380 repris in : Martin (2009), p. 199-204.

Martin, J. (1994) : « Der Wandel des Beständigen. Überlegungen zu einer historischen Anthropologie », Freiburger Universitätsblätter, 33 Heft 126, p. 35-46 ;

repris in : Winterling, A. (éd.) (2006) : Historische Anthropologie. Basistexte, Stuttgart, p. 143-157 et Martin (2009), p. 205-219.

Martin, J. (2009) : Bedingungen menschlichen Handelns in der Antike. Gesammelte Beiträge zur Historischen Anthropologie, Stuttgart.

Meier, C. (1966) : Res publica amissa. Eine Studie zu Verfassung und Geschichte der späten römischen Republik, Stuttgart ; $2^{\mathrm{e}}$ éd., Francfort-sur-le-Main, 1980.

Meier, C. (1978) : « Fragen und Thesen zu einer Theorie historischer Prozesse », in : Faber, K.-G. / Meier, c. (éd.) : Historische Prozesse, Munich, p. 11-66.

Meier, C. (1982) : Caesar, Berlin.

Meier, C. (2008) : « Antworten », in : Bernett / Nippel / Winterling (2008), p. 259-310.

Meier, C. (2015) : « Die Ordnung der Römischen Republik », Historische Zeitschrift, 300, p. 593-697.

Meier, M. (2014) : Caesar und das Problem der Monarchie in Rom (Schriften der Philosophischhistorischen Klasse der Heidelberger Akademie der Wissenschaften, 52), Heidelberg.

Meyer, I. (2006) : Von der Vision zur Reform. Der Staat der Gesetze: Ciceros Programm einer Neuordnung der Römischen Republik, 56-51 v.Chr. (Quellen und Forschungen zur Antiken Welt, 50), Munich.

Millar, F. (1977) : The Emperor in the Roman World (31 BC-AD 337), London ; $2^{\mathrm{e}}$ éd. 1992.

Millar, F. (1998) : The Crowd in Rome in the Late Republic, Ann Arbor.

Millar, F. (2002) : Rome, the Greek World, and the East, Volume 1: The Roman Republic and the Augustan Revolution, Chapel Hill / Londres.

Mommsen, Th. (1976) : Römische Geschichte, vol. 1-3 et 5, réimpression de la 9e éd. (1902-1904) en 8 volumes, Munich.

Mommsen, Th. (1887/88) : Römisches Staatsrecht, 3 tomes en 5 vol., $3^{e}$ éd., Leipzig.

Mouritsen, H. (2001) : Plebs and Politics in the Late Roman Republic, Cambridge.

Mouritsen, H. (2017) : Politics in the Roman Republic, Cambridge. 
Nebelin, M. (2014) : « Aristokratische Konkurrenz in der späten römischen Republik.

Möglichkeitsraum - Soziale Schließung - Transformation », in : Jessen, R. (éd.) : Konkurrenz in der Geschichte. Praktiken - Werte - Institutionalisierungen, Francfort-sur-le-Main / New York, p. 141-174.

Nebelin, M. (en préparation) : Freiheit und Gewalt. Die Semantik des Politischen bei Cicero, Dresde (thèse).

Nicolet, C. (1976) : Le métier de citoyen dans la Rome républicaine, Paris ; 2e éd. 1979.

Nicolet, C. (1980) : The World of the Citizen in Republican Rome, trad. par P. S. Falla, Berkeley.

Nippel, W. (1988) : Aufruhr und »Polizei« in der römischen Republik, Stuttgart.

Nippel, W. (1995) : Public Order in Ancient Rome, Cambridge.

Pfeilschifter, R. (2002) : « Die Brüchigkeit der Rituale. Bemerkungen zum Niedergang der römischen Zensur », Klio, 84, p. 440-464.

Pina Polo, F. (1996) : Contra arma verbis. Der Redner vor dem Volk in der späten römischen Republik (HABES, 22), Stuttgart.

Raaflaub, K. (1974) : Dignitatis contentio. Studien zur Motivation und politischen Taktik im Bürgerkrieg zwischen Caesar und Pompeius (Vestigia, 20), Munich.

Rilinger, R. (1976) : Die Rolle des Wahlleiters bei den römischen Konsulwahlen von 366 bis 50 v.Chr. (Vestigia, 24), Munich.

Rilinger, R. (1982) : « Die Interpretation des Niedergangs der römischen Republik durch "Revolution" und "Krise ohne Alternative" ", Archiv für Kulturgeschichte, 64, 1982, p. 279-306 ; repris in : Rilinger, R. (2007) : Ordo und dignitas. Beiträge zur römischen Verfassungs- und Sozialgeschichte, Stuttgart, p. 123-150.

Rosillo-López, C. (2017) : Public Opinion and Politics in the Late Roman Republic, Cambridge.

Samotta, I. (2009) : Das Vorbild der Vergangenheit. Geschichtsbild und Reformvorschläge bei Cicero und Sallust (Historia Einzelschriften, 204), Stuttgart.

Schmitz, W. (2009) : « Vorwort », in : Martin (2009), p. 7-16.

Schneider, H. (1974) : Wirtschaft und Politik. Untersuchungen zur Geschichte der späten römischen Republik, Erlangen.

Schneider, H. (1977) : Die Entstehung der römischen Militärdiktatur. Krise und Niedergang einer antiken Republik, Cologne.

Stemmler, M. (2000) : « Auctoritas exempli. Zur Wechselwirkung von kanonisierten Vergangenheitsbildern und gesellschaftlicher Gegenwart in der spätrepublikanischen Rhetorik », in : Linke, B. / Stemmler, M. (éd.) : Mos maiorum. Untersuchungen zu den Formen der Identitätsstiftung und Stabilisierung in der römischen Republik (Historia Einzelschriften, 41), Stuttgart, p. 141-205.

Strasburger, H. (1931) : Concordia Ordinum. Eine Untersuchung zur Politik Ciceros, Francfort-sur-leMain / Borna-Leipzig (thèse).

Strasburger, H. (1953) : « Caesar im Urteil seiner Zeitgenossen », Historische Zeitschrift, 175, p. 225-264.

Strasburger, H. (1968) : Caesar im Urteil seiner Zeitgenossen, $2^{\mathrm{e}}$ éd., Darmstadt.

Tiersch, C. (2009) : « Politische Öffentlichkeit statt Mitbestimmung? Zur Rolle der contiones in der mittleren und späten römischen Republik », Klio, 91, p. 40-68. 
Timmer, J. (2009) : « Auseinandertreten, wenn alle einer Meinung sind - Überlegungen zur discessio », Klio, 91, p. 384-405.

Timmer, J. (2017) : Vertrauen. Eine Ressource im politischen System der römischen Republik, Francfortsur-le-Main / New York.

Tröster, M. (2013) : « Roman Politics and the Whims of the Crowd: the Plebs Contionalis Revisited ", Latomus, 72, p. 128-134.

Ungern-Sternberg, J. von (1998) : « Die Legitimitätskrise der römischen Republik », Historische Zeitschrift, 266, p. 607-624.

Veyne, P. (1976) : Le pain et le cirque. Sociologie historique d'un pluralisme politique, Paris.

Walter, U. (2003) : « Ahn Macht Sinn. Familientradition und Familienprofil im republikanischen Rom », in : Hölkeskamp, K.-J. / Rüsen, J. / Stein-Hölkeskamp, E. / Grütter, H. Th. (éd.) : Sinn (in) der Antike. Orientierungssysteme, Leitbilder und Wertkonzepte im Altertum, Mayence, p. 255-278.

Walter, U. (2004a) : Memoria und res publica. Zur Geschichtskultur im republikanischen Rom, Francfort-sur-le-Main.

Walter, U. (2004b) : « "Ein Ebenbild des Vaters”. Familiale Wiederholungen in der historiographischen Traditionsbildung der römischen Republik », Hermes, 132, p. 406-425.

Walter, U. (2009) : «Struktur, Zufall, Kontingenz? Überlegungen zum Ende der römischen Republik », in : Hölkeskamp, K.-J. / Müller-Luckner, E. (éd.) : Eine politische Kultur (in) der Krise? Die letzte Generation der römischen Republik, Munich, p. 27-51.

Walter, U. (2010) : « Patronale Wohltaten oder kriminelle Mobilisierung? Sanktionen gegen unerlaubte Wahlwerbung im spätrepublikanischen Rom », in : Grüne, N. / Slanicka, S. (éd.) : Korruption. Historische Annäherung an eine Grundfigur politischer Kommunikation, Göttingen, p. 145-166.

Walter, U. (2017) : Politische Ordnung in der römischen Republik, Enzyklopädie der griechisch-römischen Antike, Munich.

Winterling, A. (2008) : « "Krise ohne Alternative” im Alten Rom », in : Bernett / Nippel / Winterling (2008), p. 219-239.

\section{NOTES}

1. C. Meier (1966) ( $2^{\mathrm{e}}$ éd. 1980). En accord avec l'objet de ce bilan sur la recherche allemande ou germanophone, je fais très largement abstraction de la documentation issue des recherches menées dans d'autres pays.

2. Mommsen (1976), (1887/88); Gelzer (1912) (réimpression 1962); Strasburger (1931), (1953) (2 éd. 1968) ; sur Heuß, voir infra note 4.

3. Sur l'importance de Mommsen et de Gelzer dans la recherche portant sur la République romaine en général, voir, en synthèse, Jehne (2006b), p. 4-6.

4. Voir Heuß (1956), (1973).

5. Raaflaub (1974); Bruhns (1978).

6. Rilinger (1976).

7. Rilinger (1982).

8. Bruhns (2003).

9. Nippel (1988) ; voir également la version anglaise (1995) fondée sur cette analyse. 
10. Hölkeskamp (1987) ( $2^{e}$ éd. 2011). Hölkeskamp n'est pas, formellement, un élève de Meier. Il a certes étudié auprès de Meier - parmi d'autres -, mais il a préparé sa thèse sous la direction de Karl-Wilhelm Welwei, à Bochum.

11. Voir sur ce point Martin (2009) (article paru initialement en 1982) ; voir également Schmitz (2009), p. 9-10.

12. C'est la brillante formule de Martin (1994).

13. Voir par exemple Linke (1998), (2006), (2014); Harders (2010).

14. Sur les activités liées aux projets en cours d'achèvement, voir le site de l'Académie : http:// www.sklaven.adwmainz.de/index.php?id=312.

15. Alföldy (1976) ( $2^{\mathrm{e}}$ éd. 1979 et $3^{\mathrm{e}}$ éd. 1984), (2011).

16. Finley (1973), (1984) (première version anglaise et édition augmentée). Une traduction allemande est parue dès 1977 sous la forme d'un livre de poche bon marché. La troisième édition (1993) propose la version augmentée.

17. Schneider (1974) ; voir aussi sa présentation de la chute de la République romaine : Schneider (1977). Sur le développement de l'histoire économique de Rome, voir Bruhns (1997).

18. Bleicken (1955) ( $2^{e}$ éd. 1968).

19. Bleicken (1975).

20. C. Meier (2015), p. 599, note 11.

21. Lundgreen (2011). Voir aussi Lundgreen (2014).

22. Nicolet (1976) ( $2^{\mathrm{e}}$ éd. 1979 ; version américaine 1980). Veyne (1976) (version abrégée en allemand : 1988).

23. Voir Jehne (2006b), p. 12-13.

24. Bruhns / David / Nippel (1997).

25. Millar (1977).

26. Les articles de Millar sur la République, parus initialement entre 1984 et 1995, sont réunis dans Millar (2002), « Part II: The Roman Republic ». Voir aussi Millar (1998).

27. Pour une vue d'ensemble synthétique, voir Jehne (1995a).

28. Jehne (éd.) (1995).

29. Auparavant, cependant, les idées de Millar ont suscité d'âpres discussions : voir par exemple Burckhardt (1990) ; Flaig (1992), (1993), (1994) ; Hölkeskamp (1987).

30. Flaig (1992), p. 84-86. Voir ensuite Flaig (1998).

31. Sur les élections, voir Jehne (2001/2003a), (2009b), (2010), (2013b).

32. Voir en outre Hölkeskamp (2004), (2017), (2019a).

33. Flaig (2003) ; voir aussi Flaig (1997).

34. Degelmann (2018).

35. Timmer (2009).

36. Hölkeskamp (1995), (2011), (2013a), (2013b) ; Jehne (2000), (2001/2003a), (2011), (2013b) ; Flaig (1995a), (2003); Pina Polo (1996); Knopf (2018).

37. Mouritsen (2001), (2017).

38. Jehne (2006b), (2011), (2013) ; Hölkeskamp (1995), (2006), (2013a), (2013b).

39. Laser (1997) ; Tiersch (2009) ; Tröster (2013) ; Knopf (2018). C'est dans cette direction que s'orientent aussi les recherches récentes sur l'opinion publique à Rome : voir désormais RosilloLópez (2017) ; Angius (2018).

40. Voir Jehne (2001/2003b), (2009b), (2010), (2013b). Sur l'ambitus, voir Jehne (1995) ; Walter (2010) ; Beck (2019) ; Karataş (2019).

41. Flaig (1995a) ; (2003), p. 155-231.

42. Flaig (1995b) ; (2003), p. 49-68.

43. Hölkeskamp (2004), (2008), (2015); voir aussi Beck (2005).

44. Walter (2004a), (2003), (2004b) (traduit en français dans le présent numéro de Trivium, dans une version abrégée). 
45. Blösel (2000); Stemmler (2000); Kirov (2010).

46. Baltrusch (1989); Pfeilschifter (2002).

47. Itgenshorst (2005).

48. Hölkeskamp (2015).

49. Hölkeskamp (2014), (2019b).

50. Nebelin (2014).

51. C. Meier (2015).

52. C. Meier (1982).

53. C. Meier (1978)

54. Au travers de l'analyse des textes centraux de Cicéron (De legibus et De re publica) et de Salluste (Epistulae ad Caesarem) : voir Lehmann (1980); Girardet (1983); voir aussi, plus tard, Meyer (2006) ; Samotta (2009).

55. Bernett (1994). Voir aussi Jehne (2003b) ou désormais, en opposition à cette analyse, Girardet (2018). Malheureusement, la thèse de Marian Nebelin, qui présente de manière convaincante la radicalisation de la pensée de Cicéron - au point de justifier l'élimination sans merci de tous les adversaires -, n'est pas encore publiée (Nebelin [en préparation]).

56. Bernett (2008). C. Meier a réagi dans ce volume à toutes les contributions portant sur les thèmes de ses recherches ou sur ses ouvrages.

57. Winterling (2008).

58. Blösel (2011), (2016). Malheureusement, l'analyse globale de Blösel n'est pas encore disponible (Blösel [en préparation]).

59. Ungern-Sternberg (1998).

60. Walter (2009).

61. Walter (2017).

62. Contre cette analyse, voir par exemple Jehne (2008), (2009a) (en référence au concept de «processus historiques » proposé par Meier : voir également Jehne [1987]).

63. M. Meier (2014). Voir aussi, prochainement, Jehne (2020 [sous presse]).

64. Timmer (2017).

65. Klingenberg (2012).

66. Künzer (2016) ; Blochmann (2017).

67. Kröss (2017).

68. Voir, provisoirement, Lundgreen (2014).

INDEX

Mots-clés : République romaine, introduction

Schlüsselwörter : römische Republik, Einleitung

\section{AUTEURS}

\section{MARTIN JEHNE}

Martin Jehne est professeur d'histoire ancienne à l'Université Technique de Dresde. Pour plus d'informations, voir la notice suivante. 